\title{
A Single-period OPF-based Strategy to Manage Distributed Energy Resources with Intertemporal Constraints in Low- voltage Grids
}

This paper was downloaded from TechRxiv (https://www.techrxiv.org).

\section{LICENSE}

CC BY-NC-SA 4.0

SUBMISSION DATE / POSTED DATE

$17-11-2021 / 19-11-2021$

\section{CITATION}

Zhan, Sen; Morren, Johan; Akker, Wouter van den; Molen, Anne van der; Paterakis, Nikolaos; Slootweg, Han (2021): A Single-period OPF-based Strategy to Manage Distributed Energy Resources with Intertemporal Constraints in Low-voltage Grids. TechRxiv. Preprint. https://doi.org/10.36227/techrxiv.17032490.v1

$\mathrm{DOI}$ 


\title{
A Single-period OPF-based Strategy to Manage Distributed Energy Resources with Intertemporal Constraints in Low-voltage Grids
}

\author{
Sen Zhan, Student Member, IEEE, Johan Morren, Member, IEEE, Wouter van den Akker, Member, IEEE,
} Anne van der Molen, Member, IEEE, Nikolaos G. Paterakis, Senior Member, IEEE, and J. G. Slootweg, Senior Member, IEEE

\begin{abstract}
The increasing installation of distributed energy resources (DERs) such as photovoltaics, electric vehicles, and heat pumps in residential premises causes voltage problems and asset overloading issues for distribution system operators (DSOs). To facilitate DSOs for minute-scale or second-scale grid operations facing a large amount of DERs with intertemporal constraints, this paper proposes a single-period optimal power flow (OPF)based strategy for DSOs to generate updated real and reactive power set-points to manage such devices to resolve the network issues while considering end-users' comfort (e.g. thermal comfort, travelling comfort). The strategy relies on specified priority for DER curtailment based on DERs' remaining flexibility. Fairness considerations are also included. The proposed strategy is tested on a representative low-voltage network including 195 nodes and 182 single-phase households under different DER penetration levels. It is found from the case study that the proposed strategy can resolve the network issues while maintaining end-users' comfort even at a high DER penetration level of $75 \%$. Compared to the rolling horizon control approach, the proposed strategy achieves faster computation and less memory requirement, allowing OPFbased grid operations at minute-scale or even second-scale.
\end{abstract}

Index Terms-Optimal power flow, distributed energy resources, intertemporal constraints, congestion management, voltage control, end-users' comfort, fairness, rolling horizon control

\section{NOMENCLATURE}

Sets

$\mathcal{H}, \mathcal{N}, \mathcal{E}, \Phi \quad$ Household set, node set, cable set, phase set

$\mathcal{T} \mathcal{F} \quad$ MV/LV transformer

$\mathcal{T} \quad$ Simulation period

Parameters

$(\overline{\tan \phi})^{E V} \quad$ Maximum reactive to real power ratio of EVs

$(\overline{\tan \phi})^{P V} \quad$ Maximum reactive to real power ratio of PVs

$(\tan \phi)^{H P} \quad$ Fixed reactive to real power ratio of HPs

S. Zhan, J. Morren, W. van den Akker, A. van der Molen, N. G. Paterakis and J. G. Slootweg are with the Department of Electrical Engineering, Eindhoven University of Technology, 5600 MB Eindhoven, The Netherlands (e-mails: s.zhan@tue.nl; j.morren@tue.nl; w.f.v.d.akker@tue.nl; a.e.v.d.molen@tue.nl; n.paterakis@tue.nl; j.g.slootweg@tue.nl).

J. Morren and J. G. Slootweg are also with the Department of Asset Management, Enexis, 5223 MB 's-Hertogenbosch, The Netherlands.

W. van den Akker is also with the Innovation Department, Alliander, 6812 AH Arnhem, The Netherlands.

A. van der Molen is also with the Grid Strategy Department, Stedin, 3011 TA Rotterdam, The Netherlands.

This work was funded by TKI Energie from the 'Toeslag voor Topconsortia voor Kennis en Innovatie (TKI)' from the ministry of Economical Affairs and Climate, under reference 1821401.

$\Delta E_{t, j, \phi}^{E V}$
$\Delta t_{t}$
$\eta_{j, \phi}^{E V}$
$\bar{i}_{i j, \phi}$
$\bar{P}_{j, \phi}^{H P}$
$\bar{S}^{t f f}$
$\bar{S}_{j, \phi}^{E V / P V}$
$\theta_{\phi}$
$\frac{\underline{v}, \bar{v}}{v_{j, \phi}^{r}}, \widehat{v_{j, \phi}^{i}}$
$c_{(t,) j, \phi}^{P V / E V / H P}$
$c_{j, \phi}^{Q}$

$C_{j, \phi}^{\operatorname{tank}}$

\section{$C O P_{j, \phi}^{H P}$ \\ $f_{t, j, \phi}^{E V / H P}$}

$p_{j, \phi}^{H P, \max }$

$p_{j, \phi}^{B L}, q_{j, \phi}^{B L}$

$p_{j, \phi}^{E V, \text { set }}$

$p_{j, \phi}^{H P, s e t}$

$p_{j, \phi}^{P V, s e t}$

$r_{i j}^{\phi_{1} \phi_{2}}, x_{i j}^{\phi_{1} \phi}$

$T_{\text {min }}$

$T_{t, j, \phi}$

$v_{\text {ref }}^{\text {mag }}$

Variables

$\Delta_{j, \phi}$

$i_{i j}^{r}, i_{i j}^{i}$
Remaining EV energy demand at time step $\mathrm{t}$ node $\mathrm{j}$ phase $\phi(\mathrm{MWh})$

Remaining EV charging time at time step $\mathrm{t}(\mathrm{h})$

EV charging efficiency at node $\mathrm{j}$ phase $\phi$

Current limit for cable from node $\mathrm{i}$ to node $\mathrm{j}$ for phase $\phi(\mathrm{kA})$

HP capacity at node $\mathrm{j}$ phase $\phi(\mathrm{MW})$

$\mathrm{MV} / \mathrm{LV}$ transformer rating (MVA)

$\mathrm{EV} / \mathrm{PV}$ inverter apparant power rating at node $\mathrm{j}$ phase $\phi$ (MVA)

Reference voltage angle for phase $\phi(\mathrm{rad})$

Minimum/maximum voltage magnitude $(\mathrm{kV})$

Real/imaginary parts of initialized voltage at node $\mathrm{j}$ phase $\phi(\mathrm{kV})$

Penalty terms for real power curtailment of $\mathrm{PVs} / \mathrm{EV}$ /HPs at (time step $\mathrm{t}$ ) node $\mathrm{j}$ phase $\phi$

Penalty term for reactive power use of PVs/EVs at node $\mathrm{j}$ phase $\phi$

Thermal capacitance of water tank at node $\mathrm{j}$ phase $\phi\left(\mathrm{MWh} /{ }^{\circ} \mathrm{C}\right)$

COP of heat pump at node $\mathrm{j}$ phase $\phi$

$\mathrm{EV} / \mathrm{HP}$ remaining flexibility at time step t node j phase $\phi(\mathrm{MWh})$

Maximum HP consumption to avoid reaching upper water tank temperature limit at node $\mathrm{j}$ phase $\phi(\mathrm{MW})$

Real/reactive power of base load consumption at node $\mathrm{j}$ phase $\phi$ (MW, MVAr)

EV charge schedule at node $\mathrm{j}$ phase $\phi(\mathrm{MW})$

HP usage schedule at node $\mathrm{j}$ phase $\phi(\mathrm{MW})$

PV generation forecast at node $\mathrm{j}$ phase $\phi(\mathrm{MW})$

Resistance/reactance of cable from node $i$ to node $\mathrm{j}$ between phase $\phi_{1}$ and phase $\phi_{2}(\Omega)$

Minimum water tank temperature $\left({ }^{\circ} \mathrm{C}\right)$

Water tank temperature at time step $\mathrm{t}$ node $\mathrm{j}$ phase $\phi\left({ }^{\circ} \mathrm{C}\right)$

Reference voltage magnitude $(\mathrm{kV})$

Weighted sum of aggregated household power curtailment at node $\mathrm{j}$ phase $\phi$ (MW)

Real/imaginary parts of current flow phasors from node $\mathrm{i}$ to node $\mathrm{j}(\mathrm{kA})$ 
$i_{\phi}^{r, t f}, i_{\phi}^{i, t f}$

Real/imaginary parts of current injection phasors from MV/LV transformer for phase $\phi(\mathrm{kA})$

$i_{j, \phi}^{r, B L}, i_{j, \phi}^{i, B L}$

$i_{j, \phi}^{r, D}, i_{j, \phi}^{i, D}$

$i_{j, \phi}^{r, E V}, i_{j, \phi}^{i, E V}$

$i_{j, \phi}^{r, H P}, i_{j, \phi}^{i, H P}$

$i_{j, \phi}^{r, P V}, i_{j, \phi}^{i, P V}$

$p_{j, \phi}^{E V}, q_{j, \phi}^{E V}$

$p_{j, \phi}^{H P}, q_{j, \phi}^{H P}$

$p_{j, \phi}^{P V}, q_{j, \phi}^{P V}$

$v_{i, \phi}^{r}, v_{i, \phi}^{i}$

Real/imaginary parts of current phasors for base load consumption at node $\mathrm{j}$ phase $\phi(\mathrm{kA})$

Real/imaginary parts of current flow phasors into node $\mathrm{j}$ phase $\phi(\mathrm{kA})$

Real/imaginary parts of current phasors for EV consumption at node $\mathrm{j}$ phase $\phi(\mathrm{kA})$

Real/imaginary parts of current phasors for HP consumption at node $\mathrm{j}$ phase $\phi(\mathrm{kA})$ generation at node $\mathrm{j}$ phase $\phi(\mathrm{kA})$

Real/reactive power consumption of $\mathrm{EV}$ at node $\mathrm{j}$ phase $\phi$ (MW, MVAr)

Real/reactive power consumption of HP at node j phase $\phi$ (MW, MVAr)

Real/reactive power generation of $\mathrm{PV}$ at node $\mathrm{j}$ phase $\phi$ (MW, MVAr)

Real/imaginary parts of voltage phasors at node
Real/imaginary parts of current phasors for PV i phase $\phi(\mathrm{kV})$

\section{INTRODUCTION}

$\mathbf{L}$ OW-VOLTAGE (LV) power grids are experiencing a transformation driven by decarbonization and decentralization. On the supply side, distributed generators such as photovoltaics (PVs) are increasingly connected to residential rooftops. While on the demand side, electric vehicles (EVs) and heat pumps (HPs) are deployed in residential premises to replace their carbon-based alternatives. The simultaneous PV injection, EV charging, and HP consumption bring considerable operational issues to distribution system operators (DSOs), such as overloading grid assets and violating the statutory voltage limits [1], [2].

Unlike medium-voltage grids where distributed generators, capacity banks, network switches, and on-load tap changers are available for system operations, DSOs for LV grids have far less controllability over grid assets traditionally. Therefore, they have to resolve these issues by reinforcing the grids. However, this process takes a long time and is costly. Fortunately, the recent roll-out of information and communication technology (ICT) infrastructures such as smart meters enables technically DSOs to engage in two-way communications with end-users to make full use of flexibility from their distributed energy resources (DERs) to address these issues.

Various studies have discussed exploiting DER flexibility for grid management. Considering allocation of power for EV charging, different charging time zones and a maximum sensitivity selection algorithm were proposed to prioritize EVs with the minimal impact on power losses [3]. The authors in [4] proposed a centralized control architecture to determine acceptance of a charging request based on a valley filling approach and a power flow engine. While in [5], the authors proposed a corrective and preventive approach to disconnect and reconnect EVs respectively based on voltage and current sensor measurement. The proposed approaches [3]-[5] effectively mitigated their network problems. However, such heuristic methods become more complicated when additional flexibility resources such as HPs are available for dispatch. Moreover, unlike mathematical optimization approaches, optimality of the proposed heuristic approaches is hard to quantify. In this regard, optimal power flow (OPF)-based models have been widely developed in the literature. An extensive OPF framework concerning various controllable assets for smart LV networks has been developed in [6]. Issues with the practical implementation of OPF-based set-points have been discussed and addressed in [7].

Using OPF-based approaches, an optimal inverter dispatch problem was proposed in [8], which systematically determines active and reactive power set-points of critical PV inverters. Binary PV-inverter selection variables were relaxed using a sparsity-promoting regularization approach, while semidefinite relaxation was leveraged to characterize the non-linear power flow relations. The follow-up studies [9]-[11] presented respectively, its risk-aware, robust, and decentralized versions. Taking into account phase unbalance for LV networks, a multiobjective OPF problem was formulated to dispatch residential PVs, aiming to improve voltage profiles while minimizing network losses and operational costs [12]. Considering mediumvoltage grid management, an OPF-based co-optimization of medium-voltage network assets and PV inverters was proposed for voltage positioning, focusing on deriving a convex but network admissible formulation [13]. However, these studies do not include devices with intertemporal constraints such as EVs and HPs, which are increasingly installed in residential premises. In such cases, a single-period OPF approach is already sufficient to derive the corresponding optimal setpoints. The optimal dispatch of energy storage systems was presented in [14] to minimize power losses in an unbalanced distribution network. However, intertemporal constraints were dropped in the single-period formulation.

Considering DERs with intertemporal constraints, rolling horizon control (RHC) approaches have been proposed in the literature. In a rolling fashion, a multi-period optimization problem is solved while only set-points for the first time step are implemented. In [15], a one-hour ahead forecast of renewable generation and demand was used for the minutescale operation of an inverter-interfaced energy storage device in an unbalanced distribution feeder. Active power set-point of the energy storage obtained from the relaxed network model was fed into a nonlinear program that exactly characterizes the power flow relations for reactive power optimization. In [16], a joint optimization of the on-load tap changer, PVs, EVs, and energy storage was formulated using the RHC approach. To reduce computational burden, a lower granularity was chosen except for the first time step. In [17], a coordinated voltage regulation strategy for MV networks considering distributed generation, energy storage, and the on-load tap changer was formulated based on the RHC approach utilizing voltage sensitivities. Both a preventive and a corrective mode were designed according to operating conditions. However, such approaches require a longer forecast horizon and more active involvement of end-users (which remains a significant challenge [18], [19]) as new set-points need to be implemented throughout the day even though the intended loading from end-users is not resulting in network issues at the exact time step (e.g. to 
compensate for future load shedding). Moreover, it presents aggravated scalability issues when already dealing with a large amount of DERs and phase unbalance.

Another group of studies that implicitly control devices with intertemporal constraints using a single-period OPF setup to ensure network integrity (current within maximum loading, voltage within statutory limits) proposed the concept of operating envelope [20], [21], which is defined as a dynamic real power import or export limit for each prosumer for each control step. Set-points of each device are left to end-users while DSOs only impose limits concerning aggregated active power import or export. As the operating envelope only considers active power limits, potential benefits from controlling reactive power are not fully explored. Furthermore, if endusers do not fully utilize the operating envelope, additional voltage problems might be incurred due to the unbalanced nature of $\mathrm{LV}$ grids.

To this point, a new research question emerges: How can DSOs manage various DERs with intertemporal constraints to ensure network integrity using a single-period optimization setup (i.e. without a multi-period ahead optimization setup) while taking end-users' comfort into consideration? To address this research question, this paper proposes a workflow for DSOs to identify and resolve network issues using a tight interaction between a power flow engine and a single-period OPF engine. The contributions of this study are three-fold:

- A single-period OPF-based model is proposed to manage DERs with intertemporal constraints based on specified curtailment priority that considers end-users' comfort, which also incorporates fairness considerations.

- A trade-off strategy for DER curtailment is developed based on their remaining flexibility, which can be used to determine the DER curtailment priority used in the OPF formulation.

- A fair comparison between the proposed strategy with a multi-period rolling horizon control strategy is performed concerning network integrity, end-users' comfort, and computational performance.

The remainder of the paper is structured as follows: Section II introduces the convex OPF model and workflow for grid operations. Section III describes a case study based on a representative LV network, while results are presented in Section IV. Section V draws conclusions for this work.

\section{MOdEl Formulation}

\section{A. DSO Workflow}

This section presents the DSO workflow and the threephase OPF formulation in detail. It is assumed that a central platform and communication links exist where end-users can send their intended schedule and the DSO can return their updated DER set-points. As shown in Fig. 1, at each time step, the DSO receives intended schedules from end-users for their various devices. When such schedules are not available due to communication failures, forecast results using historical data and real-time weather conditions can also be used. The DSO then executes a power flow program to check grid states. If grid assets are overloaded or the statutory voltage limits

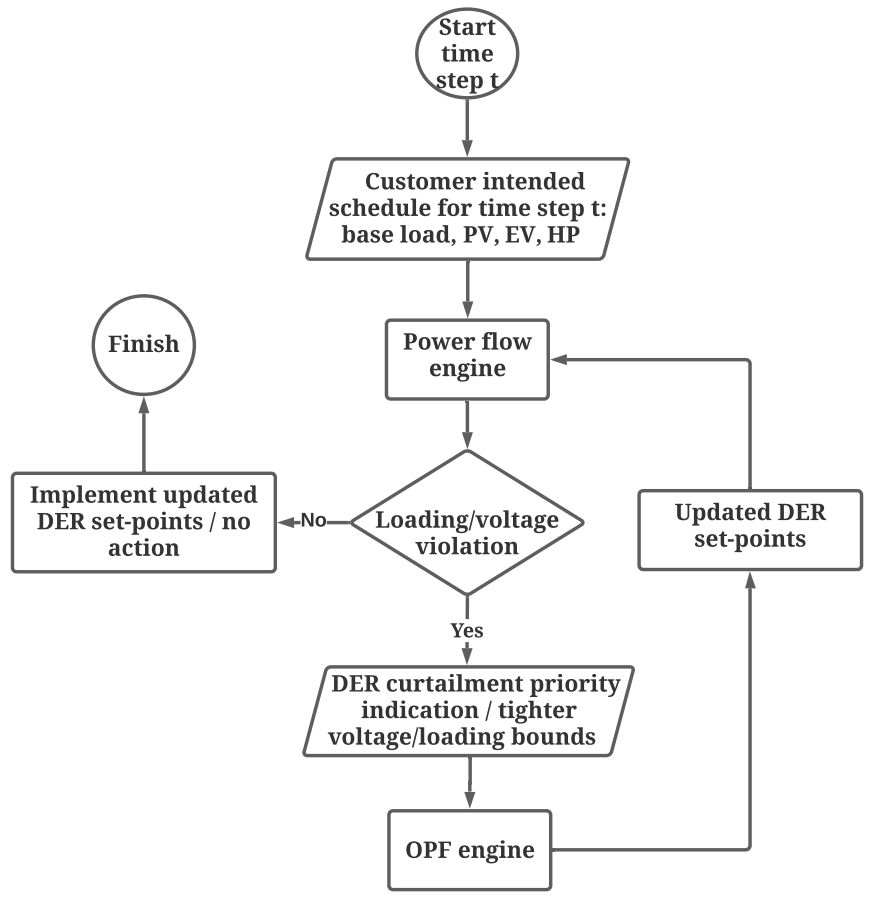

Fig. 1. DSO workflow for grid operations at each time step.

are violated, the DSO utilizes the optimal power flow engine to derive new set-points which can keep grid integrity. Right before that, the DSO collects end-users' DER curtailment trade-off plans regarding which devices to be curtailed first when necessary. In this paper, a DER curtailment trade-off strategy based on remaining flexibility is also developed. It is envisaged that through this trade-off strategy, end-users' comfort will likely be maintained. The new set-points are fed into the power flow engine to re-check grid states as the OPF formulation incorporates necessary linearization which might affect model accuracy. If no network issue is found, the new set-points are returned to end-users for implementation. If the network issues are not fully eliminated, tighter voltage or loading bounds can be introduced into the OPF formulation. From the simulation, the developed OPF formulation already presents adequate accuracy to resolve network issues. If no network issue exists for the intended schedules from end-users, no action is taken by the DSO.

\section{B. OPF Formulation}

This section presents the OPF program used to derive new set-points for DERs. A current injection based three-phase AC OPF model [22] is adopted to characterize unbalanced LV networks. Due to non-convexity, necessary convexification [23], [24] is also employed. The optimization formulation presents a quadratic objective function (1), which is expanded in (2). The penalty term $\Delta_{j, \phi}$ comprises penalties from PV curtailment, HP adjustment (both upwards and downwards), EV curtailment, and reactive power used from EVs and PVs. The quadratic formulation ensures not only minimized curtailment, but also a fair curtailment among end-users [20]. Constraints (3) to (7) enforce the Kirchhoff's current and 
voltage laws for investigated LV networks. The node current balance constraints are imposed in (3) and (4) for real and imaginary parts of the current phasors respectively. For transformers, the current balance is shown in (5). The voltage relations are presented in (6) and (7) for neighbouring buses considering the mutual inductance between different phases. Constraints (8) to (18) concern end-users. The current balance constraints for each end-user are imposed in (8) and (9). Real and reactive power of PVs, EVs, HPs, and base load are respectively defined in (10). Constraint (11) ensures the HP consumption is within its rating and a manually imposed limit to avoid the water tank storage's temperature reaches its upper limit (when the HP is increasing its consumption to utilize PV power that would be otherwise curtailed). Constraint (12) enforces the fixed power factor for HPs. Constraint (13) to (15) pertain to EVs, where (13) ensures EV charging will not exceed their intended plan. Constraints (14) and (15) enforce respectively EVs' apparent power and power factor limits. A similar set of constraints (16) to (18) apply to PVs. Constraints (19) to (22) ensure the network integrity, where (19) pertains to the statutory voltage limits, (20) and (21) ensure cables and transformers are not overloaded. The slack bus voltage is defined in (22).

$$
\underset{p_{j, \phi}^{E V}, p_{j, \phi}^{H P}, p_{j, \phi}^{P V}, q_{j, \phi}^{E V}, q_{j, \phi}^{H P}, q_{j, \phi}^{P V}, \forall j \in \mathcal{H}, \forall \phi \in \Phi}{\operatorname{minimize}}\left\{\sum_{j \in \mathcal{H}} \sum_{\phi \in \Phi} \Delta_{j, \phi}^{2}\right\}
$$

subject to

$$
\begin{aligned}
& \Delta_{j, \phi}=c_{j, \phi}^{P V}\left(p_{j, \phi}^{P V, s e t}-p_{j, \phi}^{P V}\right)+c_{j, \phi}^{H P}\left|p_{j, \phi}^{H P, s e t}-p_{j, \phi}^{H P}\right| \\
& +c_{j, \phi}^{E V}\left(p_{j, \phi}^{E V, \text { set }}-p_{j, \phi}^{E V}\right)+c_{j, \phi}^{Q}\left(\left|q_{j, \phi}^{E V}\right|+\left|q_{j, \phi}^{P V}\right|\right), \\
& \forall j \in \mathcal{N}, \forall \phi \in \Phi \\
& \sum_{i: i \rightarrow j} i_{i j, \phi}^{r}=\sum_{k: j \rightarrow k} i_{j k, \phi}^{r}+i_{j, \phi}^{r, D}, \forall j \in \mathcal{N}, \forall \phi \in \Phi \\
& \sum_{i: i \rightarrow j} i_{i j, \phi}^{i}=\sum_{k: j \rightarrow k} i_{j k, \phi}^{i}+i_{j, \phi}^{i, D}, \forall j \in \mathcal{N}, \forall \phi \in \Phi \\
& i_{\phi}^{r, t f}=\sum_{k: j \rightarrow k} i_{j k, \phi}^{r}, i_{\phi}^{i, t f}=\sum_{k: j \rightarrow k} i_{j k, \phi}^{i}, j \in \mathcal{T} \mathcal{F}, \forall \phi \in \Phi \\
& {\left[\begin{array}{c}
v_{i, a}^{r} \\
v_{i, b}^{r} \\
v_{i, b}^{r}
\end{array}\right]-\left[\begin{array}{c}
v_{j, a}^{r} \\
v_{j, b}^{r} \\
v_{j, c}^{r}
\end{array}\right]=\left[\begin{array}{ccc}
r_{i j}^{a a} & r_{i j}^{a b} & r_{i j}^{c a} \\
r_{i j}^{a b} & r_{i j}^{b b} & r_{i j}^{b c} \\
r_{i j}^{c a} & r_{i j}^{b c} & r_{i j}^{c c}
\end{array}\right]\left[\begin{array}{c}
i_{i j, a}^{r} \\
i_{i j, b}^{r} \\
i_{i j, c}^{r}
\end{array}\right]} \\
& -\left[\begin{array}{ccc}
x_{i j}^{a a} & x_{i j}^{a b} & x_{i j}^{c a} \\
x_{i j}^{a b} & x_{i j}^{b b} & x_{i j}^{b c} \\
x_{i j}^{c a} & x_{i j}^{b c} & x_{i j}^{c c}
\end{array}\right]\left[\begin{array}{c}
i_{i j, a}^{i} \\
i_{i j, b}^{i} \\
i_{i j, c}^{i}
\end{array}\right], \forall(i, j) \in \mathcal{E} \\
& {\left[\begin{array}{c}
v_{i, a}^{i} \\
v_{i, b}^{i} \\
v_{i, b}^{i}
\end{array}\right]-\left[\begin{array}{c}
v_{j, a}^{i} \\
v_{j, b}^{i} \\
v_{j, c}^{i}
\end{array}\right]=\left[\begin{array}{ccc}
r_{i j}^{a a} & r_{i j}^{a b} & r_{i j}^{c a} \\
r_{i j}^{a b} & r_{i j}^{b b} & r_{i j}^{b c} \\
r_{i j}^{c a} & r_{i j}^{b c} & r_{i j}^{c c}
\end{array}\right]\left[\begin{array}{c}
i_{i j, a}^{i} \\
i_{i j, b}^{i} \\
i_{i j, c}^{i}
\end{array}\right]} \\
& +\left[\begin{array}{lll}
x_{i j}^{a a} & x_{i j}^{a b} & x_{i j}^{c a} \\
x_{i j}^{a b} & x_{i j}^{b b} & x_{i j}^{b c} \\
x_{i j}^{c a} & x_{i j}^{b c} & x_{i j}^{c c}
\end{array}\right]\left[\begin{array}{c}
i_{i j, a}^{r} \\
i_{i j, b}^{r} \\
i_{i j, c}^{r}
\end{array}\right], \forall(i, j) \in \mathcal{E} \\
& i_{j, \phi}^{r, D}=i_{j, \phi}^{r, B L}+i_{j, \phi}^{r, H P}+i_{j, \phi}^{r, E V}-i_{j, \phi}^{r, P V}, \forall j \in \mathcal{H}, \forall \phi \in \Phi \\
& i_{j, \phi}^{i, D}=i_{j, \phi}^{i, B L}+i_{j, \phi}^{i, H P}+i_{j, \phi}^{i, E V}-i_{j, \phi}^{i, P V}, \forall j \in \mathcal{H}, \forall \phi \in \Phi
\end{aligned}
$$

$$
\begin{aligned}
& p_{j, \phi}^{k}=v_{j, \phi}^{r} i_{j, \phi}^{r, k}+v_{j, \phi}^{i} i_{j, \phi}^{i, k}, q_{j, \phi}^{k}=v_{j, \phi}^{i} i_{j, \phi}^{r, k}-v_{j, \phi}^{r} i_{j, \phi}^{i, k}, \\
& \forall k \in P V \cup E V \cup H P \cup B L, \forall j \in \mathcal{H}, \forall \phi \in \Phi \\
& 0 \leq p_{j, \phi}^{H P} \leq \min \left(\bar{P}_{j, \phi}^{H P}, p_{j, \phi}^{H P, \max }\right), \forall j \in \mathcal{H}, \forall \phi \in \Phi \\
& q_{j, \phi}^{H P}=p_{j, \phi}^{H P}(\tan \phi)^{H P}, \forall j \in \mathcal{H}, \forall \phi \in \Phi \\
& 0 \leq p_{j, \phi}^{E V} \leq p_{j, \phi}^{E V, s e t}, \forall j \in \mathcal{H}, \forall \phi \in \Phi \\
& \left(p_{j, \phi}^{E V}\right)^{2}+\left(q_{j, \phi}^{E V}\right)^{2} \leq\left(\bar{S}_{j, \phi}^{E V}\right)^{2}, \forall j \in \mathcal{H}, \forall \phi \in \Phi \\
& -(\overline{\tan \phi})^{E V} p_{j, \phi}^{E V} \leq q_{j, \phi}^{E V} \leq(\overline{\tan \phi})^{E V} p_{j, \phi}^{E V}, \forall j \in \mathcal{H}, \forall \phi \in \Phi \\
& 0 \leq p_{j, \phi}^{P V} \leq p_{j, \phi}^{P V, \text { set }}, \forall j \in \mathcal{H}, \forall \phi \in \Phi \\
& \left(p_{j, \phi}^{P V}\right)^{2}+\left(q_{j, \phi}^{P V}\right)^{2} \leq\left(\bar{S}_{j, \phi}^{P V}\right)^{2}, \forall j \in \mathcal{H}, \forall \phi \in \Phi \\
& -(\overline{\tan \phi})^{P V} p_{j, \phi}^{P V} \leq q_{j, \phi}^{P V} \leq(\overline{\tan \phi})^{P V} p_{j, \phi}^{P V}, \forall j \in \mathcal{H}, \forall \phi \in \Phi \\
& (\underline{v})^{2} \leq\left(v_{j, \phi}^{r}\right)^{2}+\left(v_{j, \phi}^{i}\right)^{2} \leq(\bar{v})^{2}, \forall j \in \mathcal{N}, \forall \phi \in \Phi \\
& \left(i_{i j, \phi}^{r}\right)^{2}+\left(i_{i j, \phi}^{i}\right)^{2} \leq\left(\bar{i}_{i j, \phi}\right)^{2}, \forall(i, j) \in \mathcal{E}, \forall \phi \in \Phi \\
& v_{r e f}^{\operatorname{mag}}\left[\sum_{\phi \in \Phi} \sqrt{\left(i_{\phi}^{r, t f}\right)^{2}+\left(i_{\phi}^{i, t f}\right)^{2}}\right] \leq \bar{S}_{t f} \\
& v_{t f, \phi}^{r}=v_{r e f}^{\text {mag }} \cos \left(\theta_{\phi}\right), v_{t f, \phi}^{i}=v_{r e f}^{\text {mag }} \sin \left(\theta_{\phi}\right), \forall \phi \in \Phi
\end{aligned}
$$

\section{Convexification and Solution Approach}

The above OPF formulation presents a non-convex optimization problem, where non-convexity of the above model arises from constraints (10) and the left side of the constraint (19). To convexify (10), the real and imaginary parts of voltage phasors in (10) are substituted by fixed parameters (e.g. an initialization with voltage phasors of $1 \mathrm{pu}$ and $120^{\circ}$ difference), shown as below.

$$
\begin{array}{r}
p_{j, \phi}^{k}=\widehat{v_{j, \phi}^{r}} i_{j, \phi}^{r, k}+\widehat{v_{j, \phi}^{i}} i_{j, \phi}^{i, k}, q_{j, \phi}^{k}=\widehat{v_{j, \phi}^{i}} i_{j, \phi}^{r, k}-\widehat{v_{j, \phi}^{r}} i_{j, \phi}^{i, k}, \\
\forall k \in P V \cup E V \cup H P \cup B L, \forall j \in \mathcal{H}, \forall \phi \in \Phi
\end{array}
$$

For the left side of the constraint (19), a first-order Taylor expansion is used around a given value (e.g. the same initialization above), which gives a linearized formulation.

$$
\begin{aligned}
& (\underline{v})^{2} \leq\left(\widehat{v_{j, \phi}^{r}}\right)^{2}+2 \widehat{v_{j, \phi}^{r}}\left(v_{j, \phi}^{r}-\widehat{v_{j, \phi}^{r}}\right) \\
& \left.+\widehat{\left(v_{j, \phi}^{i}\right.}\right)^{2}+\widehat{2 v_{j, \phi}^{i}}\left(v_{j, \phi}^{i}-\widehat{v_{j, \phi}^{i}}\right), \forall j \in \mathcal{N}, \forall \phi \in \Phi
\end{aligned}
$$

Considering inaccuracy issues brought by the initialization, a two-stage approach [22] is used. In the first stage, the voltage parameters $\widehat{v_{j, \phi}^{r}}$ and $\widehat{v_{j, \phi}^{i}}$ are initialized as above. Alternatively, the voltage profile from the power flow engine using intended schedules from end-users could also be used. However, when the voltage is driven far away from the statutory limits, such initialization may result in an infeasible solution for the OPF problem. The optimized voltage results from the first-stage optimization are then fed as voltage parameters in (23) and (24). Following this the OPF problem is solved for the second time. Although additional stages can be explored following the same routine, we found in our experiments that such a two-stage approach already produces highly accurate results. 


\section{Trade-off Strategy of DER Curtailment}

The solution of the above OPF problem depends on selection of the penalty parameters, including $c_{j, \phi}^{P V}, c_{j, \phi}^{E V}, c_{j, \phi}^{H P}$, and $c_{j, \phi}^{Q}$ in (2). The higher penalty parameter assigned, generally, the less the corresponding DER will be curtailed. Selection of the penalty parameters, thus indicates end-users' curtailment trade-off between different DERs, affecting their comfort differently. In real life implementation, the curtailment trade-off between different DERs can be specified by end-users based on their preferences. In this study, a trade-off strategy for DER curtailment is developed based on DERs's remaining flexibility. As PV curtailment generally is not incurred simultaneously with EV curtailment (different regulating directions), the tradeoff exists only between PVs and HPs (HP consumption can be increased to absorb excess PV injection), or HPs and EVs (both can be curtailed). It is assumed for PV and HP tradeoff, PV is given priority until the water tank storage of the HP reaches the upper limit to encourage self-consumption. While for EV and HP trade-off, priority is given to the one with a lower flexibility level, which is defined as the amount of energy that can be further curtailed before the corresponding comfort is affected. Details are presented in (25)-(26) for EVs and HPs respectively, which are changing continuously over the simulation period.

$$
\begin{gathered}
f_{t, j, \phi}^{E V}=\bar{S}^{E V} \Delta t_{t},-\Delta E_{t, j, \phi}^{E V} / \eta_{j, \phi}^{E V}, \\
\forall t \in \mathcal{T}, \forall j \in \mathcal{H}, \forall \phi \in \Phi \\
f_{t, j, \phi}^{H P}=C_{j, \phi}^{t a n k}\left(T_{t, j, \phi}-T_{m i n}\right) / \mathrm{COP}_{j, \phi}^{H P}, \\
\forall t \in \mathcal{T}, \forall j \in \mathcal{H}, \forall \phi \in \Phi
\end{gathered}
$$

Following this, the penalty terms can be defined in (27). In this way, the DER with higher flexibility will be curtailed first, thus likely preserving end-users' different comfort. Moreover, use of reactive power will be incentivized.

$$
\begin{aligned}
& \begin{cases}c_{t, j, \phi}^{P V}>c_{t, j, \phi}^{H P} \geq c_{t, j, \phi}^{E V}>c_{t, j, \phi}^{Q} & \text { if } f_{t, j, \phi}^{E V} \geq f_{t, j, \phi}^{H P}, \\
c_{t, j,}^{P V}>c_{t, j} \geq c_{t, j, \phi}^{H P}>c_{t, j, \phi}^{Q} & \text { otherwise }\end{cases} \\
& \forall t \in \mathcal{T}, \forall j \in \mathcal{H}, \forall \phi \in \Phi
\end{aligned}
$$

\section{CASE STUdY}

\section{A. Case Description}

1) Test Network: The simulation is performed on a representative Dutch LV network with 195 nodes. The network topology and load data are made publicly available on 4TU.ResearchData [25]. Through 5 feeders extending from the MV/LV transformer, 182 single-phase residential endusers are connected, randomly spreading over different phases. As shown in Fig. 2, the network buses are marked with blue dots, while black arrows show where the end-users are connected. The yellow square indicates the secondary side of the MV/LV transformer as the slack bus with a voltage magnitude of $1 \mathrm{pu}$. The statutory voltage limits are set as 1.04 pu maximum and $0.96 \mathrm{pu}$ minimum at the buses. Connecting cables include Al-95mm, Al-150mm, Cu- $6 \mathrm{~mm}$, and $\mathrm{Cu}-35 \mathrm{~mm}$. Distance between most neighbouring buses ranges from 4 to 15 meters. The MV/LV transformer has a maximum loading of

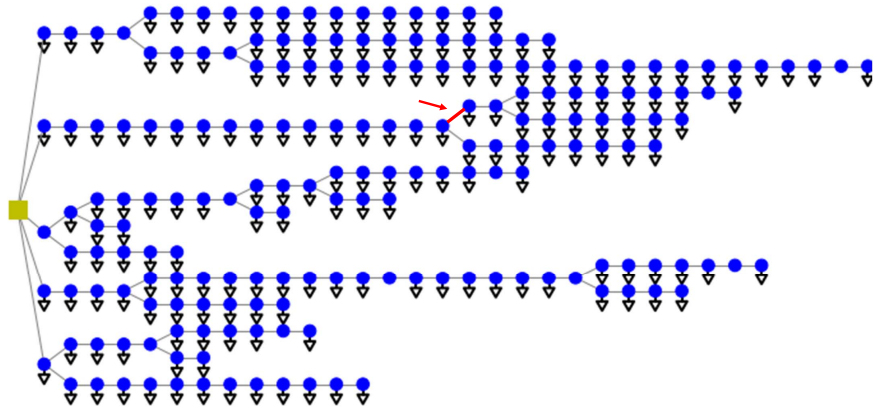

Fig. 2. Representative Dutch LV network topology. Buses are marked with blue dots, while single-phase end-users are marked with black arrows. The LV side of MV/LV transformer is marked with the yellow square. A 120-meter $\mathrm{Cu}-35 \mathrm{~mm}$ cable is marked with a red line which gives rise to frequent voltage problems for downstream end-users.

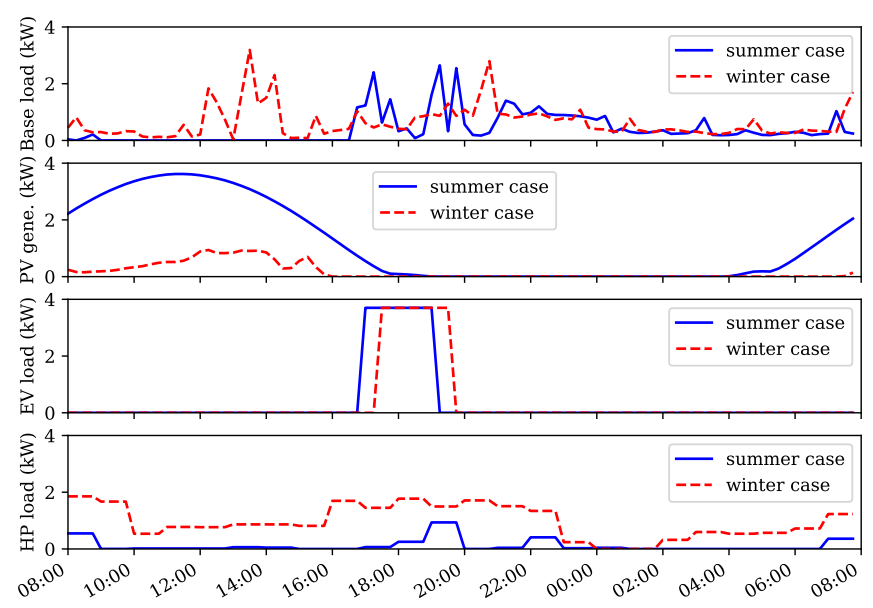

Fig. 3. Base load, PV, EV, and HP power profiles for a sample household in the summer and winter test cases.

$630 \mathrm{kVA}$. A 120-meter $\mathrm{Cu}-35 \mathrm{~mm}$ cable giving rise to frequent voltage problems for downstream end-users is marked with a red line in Fig. 2.

2) DER Specifications: Three DER penetration scenarios are considered for this case study: 50\%, 75\%, and $100 \%$, which are defined as the percentage of end-users with controllable DERs. The simulation is performed with a $15-\mathrm{min}$ resolution given the granularity of available data, though a higher resolution (e.g. 5-min, 1-min) could have been achieved given the computational speed of the power flow and OPF programs. The base load profiles are based on anonymous smart meter data from a Dutch DSO, while the PV generation profile is calculated from an annual solar irradiance dataset from Solcast [26] assuming a 4.5kW PV (3.75 kVA inverter) installation for each household. The HP consumption profiles are based on anonymous smart meter gas usage data from a Dutch DSO, assuming 70\% of the gas is used for domestic heating. The HPs are assumed to work at a COP of 3 and directly heat the water tanks, which will then supply domestic hot water and space heating use. The water tank is assumed to be $1000 \mathrm{~L}$ for households with no more than $5 \mathrm{~kW}$ heating demand, while an additional 200L is assumed for each $\mathrm{kW}$ above 5kW. For EV charging, the Monto Carlo simulation is 
performed by assuming normally distributed EV arrival time and fixed electricity demand of $7.5 \mathrm{kWh}$ per day. A $230 \mathrm{~V}, 16 \mathrm{~A}$ charger with a $90 \%$ efficiency is assumed for residential EV charging. The EV departure time is assumed the same for all end-users to be the end of the simulation period, which lasts from 8:00 am to 8:00 am the next day. The assumption ensures the 1-day simulation period incorporates a full charging cycle for each EV so that EV users' comfort levels can be adequately assessed. However, the proposed strategy is generally applicable, requiring no assumption made above for its implementation by DSOs. For illustration purposes, base load, PV, EV, and HP profiles for a sample household for the summer case and the winter case are presented in Fig. 3, where the simultaneous PV injection during the summer day as well as the uncontrolled EV charging and HP consumption during the winter day could result in problems such as violating the statutory voltage limits and overloading grid assets.

3) End-users' Comfort: End-users' comfort is evaluated for EVs, HPs, and PVs. For EVs, the state of charge at the end of the simulation is seen as the key indicator for comfort evaluation. If the EV is fully charged, then the end-user does not experience any discomfort. For HPs, the temperature of the connecting water tank storage which supplies hot water for domestic use and space heating is assumed as the key indicator. If the temperature is within the upper and lower bounds, the end-users' comfort is not affected. For the case study, the upper bound for temperature is assumed to be $55^{\circ} \mathrm{C}$, and the lower bound is assumed as $40^{\circ} \mathrm{C}$ [27]. For the summer test case, the water tank storage is assumed to be normally operated at $47.5^{\circ} \mathrm{C}$ (if no control action from the DSO is implemented), giving it possibility for bidirectional control (e.g. absorbing extra PV power or curtailing power when network congestion occurs). While for the winter case, the water tank storage is assumed to be normally operated at its upper limit of $55^{\circ} \mathrm{C}$, giving it full potential to sustain curtailment. For PVs, there is no such technical indicator. It is assumed if combined PV curtailment for each end-user over the simulation period is less than $30 \%$, PV users' comfort is met.

4) Trade-off Parameters:

$$
\begin{array}{r}
\left\{\begin{aligned}
c_{t, j, \phi}^{E V}=1, c_{t, j, \phi}^{H P}=0.8 & \text { if } f_{t, j, \phi}^{E V} \leq f_{t, j, \phi}^{H P} \\
c_{t, j, \phi}^{E V}=0.8, c_{t, j, \phi}^{H P}=1 & \text { otherwise }
\end{aligned}\right. \\
\forall t \in \mathcal{T}, \forall j \in \mathcal{H}, \forall \phi \in \Phi
\end{array}
$$

The penalty parameters for real power curtailment are set close to one so that a certain level of fairness will be ensured through the quadratic objective function (1) concerning aggregated real power curtailment for a household, while that of reactive power curtailment is set to one magnitude lower to incentivize reactive power use. Specially, the PV and reactive power penalty parameters are assigned such that $c_{j, \phi}^{P V}=1.2$, $c_{j, \phi}^{Q}=0.1$, which are constants so that PVs will be given priority, and reactive power use will be encouraged. The EV and HP curtailment penalties are defined in (28) such that the one with a lower amount of flexibility will be given higher priority. It is envisaged in this way both end-users's thermal and travelling comfort will be maintained. The parameters are not assigned as e.g. 0.99/1.01 which are closer to one because of reactive power impact from HPs (as reactive power curtailment are accompanied by active power curtailment for HPs, additional benefits from reactive power curtailment concerning e.g. reducing asset loading and increasing voltage might override the 0.02 difference in penalty terms).

The proposed single-period OPF is formulated with the Gurobi [28] Python interface and solved to optimality using a laptop with an Intel Core i5-9300H CPU running at $2.4 \mathrm{GHz}$ with an 8GB RAM. The power flow program is modelled with the Python Pandapower package [29].

\section{RESULTS}

This section introduces key results found from simulations on the case study. First, results from the two case studies including a summer case with a high PV generation and a winter case with a high HP consumption which coincides with EV load during the night are elaborated, concerning network issues and end-users' comfort. Following this, advantages of the proposed DER curtailment trade-off strategy is explored compared with the static penalty parameters either giving EVs or HPs full priority. Then the fairness consideration from the quadratic objective function is introduced. Finally, the proposed strategy is compared with the rolling horizon control approach to explore its benefit and loss.

\section{A. Summer Case}

From Fig. 4 and the transformer loading in Fig. 5, the uncontrolled PV injection during the day and the simultaneous EV charging during the early evening result in two voltage and loading peaks. When uncontrolled, voltage goes beyond both the lower and upper limits, while cables and the transformer are overloaded during the evening. Using the proposed OPFbased approach to generate new DER set-points, the voltage peaks and the extra loading of cables and the transformer are fully removed, thus maintaining the grid integrity. From HP consumption and PV generation profiles in Fig. 5, it can be observed that HP consumption has been increased to absorb PV power that would otherwise be curtailed, which is the result of the PV/HP trade-off setting.

Impacts of the optimized control decisions on end-users' comfort for the 3 different DER penetration levels are summarized in Table I. It can be seen that for PVs, the maximum curtailment is $4.2 \mathrm{kWh}$ under the $100 \%$ DER penetration scenario, which takes up $15 \%$ of the expected generation and is lower than the set $30 \%$ PV curtailment limit. For HPs, whose consumption has been increased to absorb PV power, the upper temperature limit of $55^{\circ} \mathrm{C}$ has been kept. For EV users, the departure state of charge is all $100 \%$, indicating their use of EVs has not been affected. It can be summarized that for the summer case, the proposed model can keep the network integrity while maintaining end-users' comfort under all DER penetration scenarios.

\section{B. Winter Case}

For the winter case, the voltage peak and the loading peaks for cables and the transformer are seen during the early 

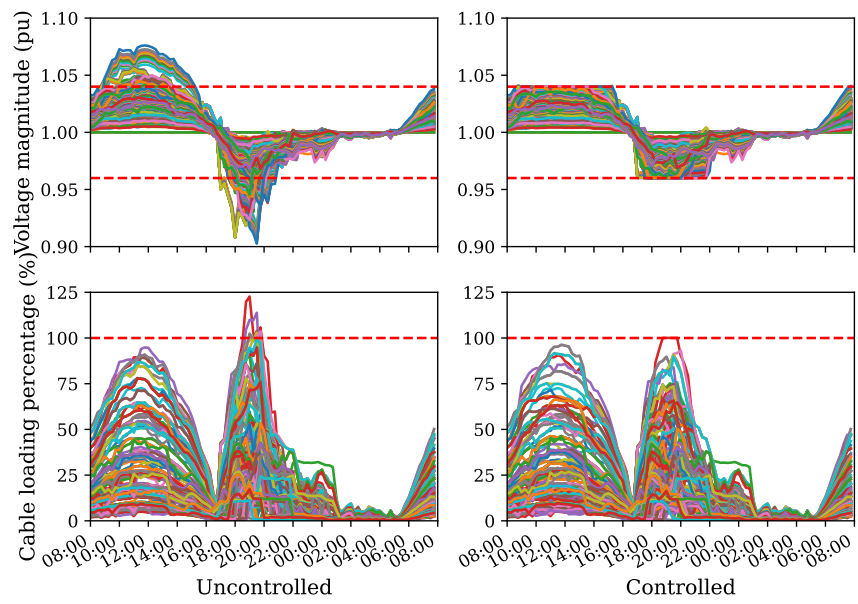

Fig. 4. Voltage/cable loading under summer case at $100 \%$ DER penetration. Lines correspond to individual buses and cables, respectively.
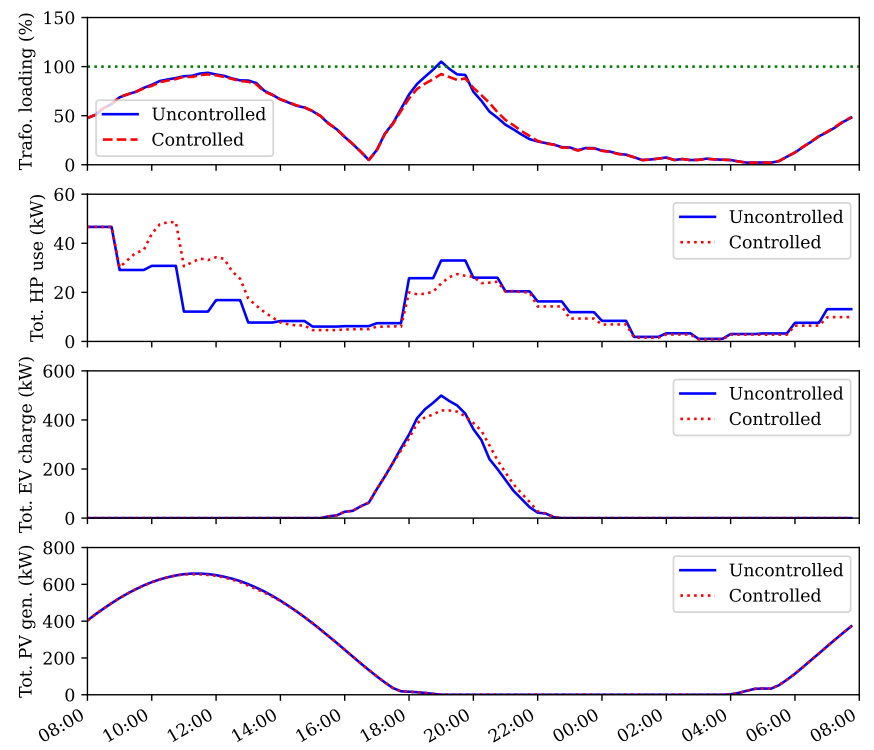

Fig. 5. Transformer loading, total HP consumption, EV charge, and PV generation under summer case at $100 \%$ DER penetration.

evening, but at a higher level than that of the summer case due to HP consumption for house heating and hot water use. The proposed approach still manages to keep the network integrity as portrayed in Fig. 6 and transformer loading profiles in Fig. 7. From Fig. 7, the curtailed HP and EV demand during the early evening is gradually compensated over the night, which is constrained by the statutory lower voltage limit as can be seen in Fig. 6. Moreover, EVs have experienced larger amount of curtailment compared to HPs due to the HP/EV trade-off setting. As EV charge can be delayed for a long period while still being fully charged upon departure, EVs generally have higher amount of flexibility and thus lower penalties in the OPF formulation.

Impacts on end-users' comfort are summarized in Table II. For PV users, curtailment is negligible, which occurs due to the phase unbalance and numerical accuracy issues brought from the Gurobi optimization engine. For HP users, impacts
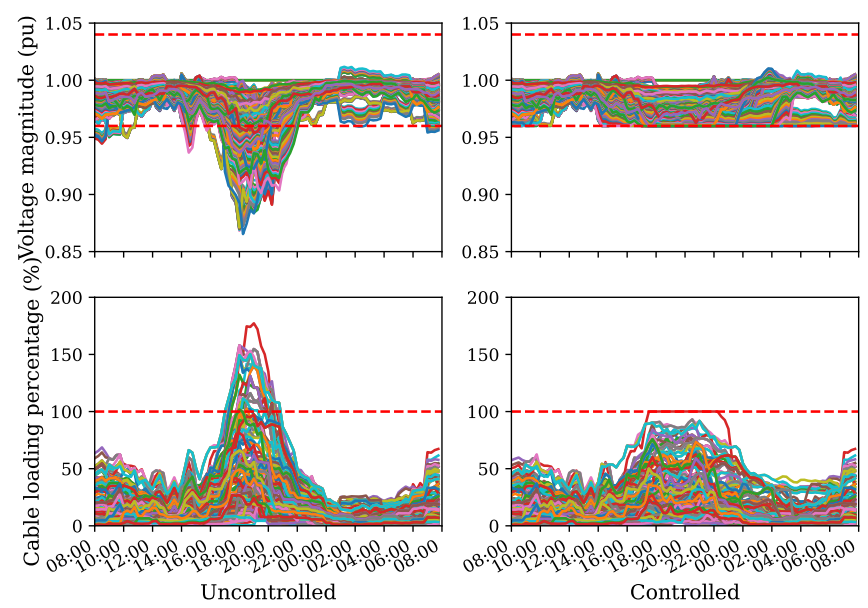

Fig. 6. Voltage/cable loading under winter case at $100 \%$ DER penetration. Lines correspond to individual buses and cables, respectively.
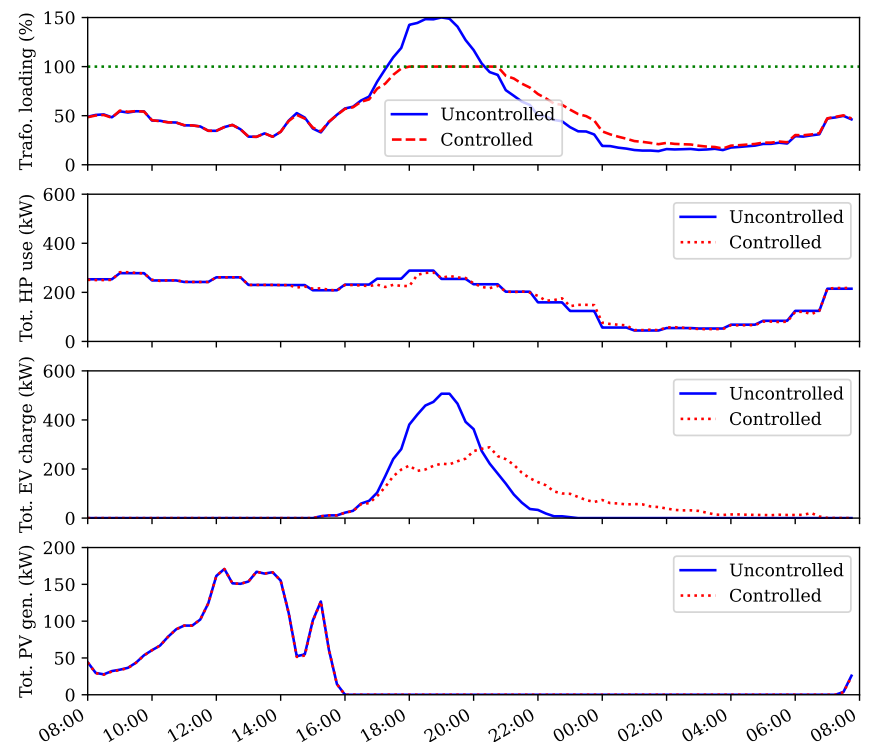

Fig. 7. Transformer loading, total HP consumption, EV charge, and PV generation under winter case at $100 \%$ DER penetration.

are not seen until the extreme loading condition of $100 \%$ DER penetration, where the water tank temperature drops below the lower limit of $40^{\circ} \mathrm{C}$ to $36.2^{\circ} \mathrm{C}$. Despite such a high loading, the average water tank temperature is over $53.3^{\circ} \mathrm{C}$, with only 2 out of 182 end-users going beyond the lower limit (by $0.1^{\circ} \mathrm{C}$ and $3.8^{\circ} \mathrm{C}$ respectively), thus their thermal comfort has been compromised. In such case, additional compensation can be delivered to the affected end-users. For EV users, the minimum state of charge at the end of the simulation is more than $99.1 \%$ under all scenarios, which indicates that EV users' comfort has been maintained.

\section{DER Curtailment Trade-off}

This section presents benefits brought from the DER curtailment trade-off strategy in (25) - (27). Two persistency-based cases are compared, in which EVs and HPs are respectively given full priority over each other. In the EV-priority case, 
TABLE I

END-USERS' COMFORT FOR SUMMER CASE

\begin{tabular}{lcccccc}
\hline & Min & Q2 & Median & Q4 & Max & Mean \\
\hline Tot. PV Curt.(kWh)-50\% & 0.00 & 0.00 & 0.00 & 0.00 & 0.05 & 0.00 \\
Tot. PV Curt.(kWh)-75\% & 0.00 & 0.00 & 0.00 & 0.00 & 0.05 & 0.01 \\
Tot. PV Curt.(kWh)-100\% & 0.00 & 0.00 & 0.00 & 0.02 & $\mathbf{4 . 2 1}$ & 0.08 \\
\hline Max Tank Temp. $\left({ }^{\circ} \mathrm{C}\right)-50 \%$ & 47.50 & 47.50 & 47.50 & 47.50 & 51.93 & 47.58 \\
Max Tank Temp. $\left({ }^{\circ} \mathrm{C}\right)-75 \%$ & 47.50 & 47.50 & 47.50 & 47.51 & 54.15 & 47.65 \\
Max Tank Temp. $\left({ }^{\circ} \mathrm{C}\right)-100 \%$ & 47.50 & 47.50 & 47.50 & 47.69 & 55.00 & 48.34 \\
\hline Depart. EV SOC-50\% & $100.0 \%$ & $100.0 \%$ & $100.0 \%$ & $100.0 \%$ & $100.0 \%$ & $100.0 \%$ \\
Depart. EV SOC-75\% & $100.0 \%$ & $100.0 \%$ & $100.0 \%$ & $100.0 \%$ & $100.0 \%$ & $100.0 \%$ \\
Depart. EV SOC-100\% & $100.0 \%$ & $100.0 \%$ & $100.0 \%$ & $100.0 \%$ & $100.0 \%$ & $100.0 \%$ \\
\hline
\end{tabular}

TABLE II

END-USERS' COMFORT FOR WINTER CASE

\begin{tabular}{lcccccc}
\hline & Min & Q2 & Median & Q4 & Max & Mean \\
\hline Tot. PV Curt.(kWh)-50\% & 0.00 & 0.00 & 0.00 & 0.00 & 0.08 & 0.00 \\
Tot. PV Curt.(kWh)-75\% & 0.00 & 0.00 & 0.00 & 0.00 & 0.09 & 0.01 \\
Tot. PV Curt.(kWh)-100\% & 0.00 & 0.00 & 0.00 & 0.01 & 0.13 & 0.01 \\
\hline Min Tank Temp. $\left({ }^{\circ} \mathrm{C}\right)-50 \%$ & 47.61 & 54.93 & 55.00 & 55.00 & 55.00 & 54.75 \\
Min Tank Temp. $\left({ }^{\circ} \mathrm{C}\right)-75 \%$ & 43.75 & 54.30 & 55.00 & 55.00 & 55.00 & 54.38 \\
Min Tank Temp. $\left({ }^{\circ} \mathrm{C}\right)-100 \%$ & $\mathbf{3 6 . 1 7}$ & 53.41 & 54.49 & 54.92 & 55.00 & 53.37 \\
\hline Depart. EV SOC-50\% & $100.0 \%$ & $100.0 \%$ & $100.0 \%$ & $100.0 \%$ & $100.0 \%$ & $100.0 \%$ \\
Depart. EV SOC-75\% & $99.1 \%$ & $100.0 \%$ & $100.0 \%$ & $100.0 \%$ & $100.0 \%$ & $100.0 \%$ \\
Depart. EV SOC-100\% & $99.6 \%$ & $100.0 \%$ & $100.0 \%$ & $100.0 \%$ & $100.0 \%$ & $100.0 \%$ \\
\hline
\end{tabular}

TABLE III

END-USERS' COMFORT FOR WINTER CASE UNDER EV-PRIORITY SETTING

\begin{tabular}{lcccccc}
\hline & Min & $\mathrm{Q} 2$ & Median & $\mathrm{Q} 4$ & Max & Mean \\
\hline Tot. PV Curt.(kWh)-50\% & 0.00 & 0.00 & 0.00 & 0.00 & 0.06 & 0.00 \\
Tot. PV Curt.(kWh)-75\% & 0.00 & 0.00 & 0.00 & 0.00 & 0.06 & 0.00 \\
Tot. PV Curt.(kWh)-100\% & 0.00 & 0.00 & 0.00 & 0.01 & 0.09 & 0.01 \\
\hline Min Tank Temp. $\left({ }^{\circ} \mathrm{C}\right)-50 \%$ & $\mathbf{3 2 . 6 7}$ & 54.64 & 55.00 & 55.00 & 55.00 & 53.94 \\
Min Tank Temp. $\left({ }^{\circ} \mathrm{C}\right)-75 \%$ & $\mathbf{3 1 . 5 8}$ & 51.35 & 54.91 & 55.00 & 55.00 & 52.28 \\
Min Tank Temp. $\left({ }^{\circ} \mathrm{C}\right)-100 \%$ & $\mathbf{2 1 . 3 8}$ & 43.54 & 48.85 & 52.12 & 55.00 & 46.69 \\
\hline Depart. EV SOC-50\% & $100.0 \%$ & $100.0 \%$ & $100.0 \%$ & $100.0 \%$ & $100.0 \%$ & $100.0 \%$ \\
Depart. EV SOC-75\% & $100.0 \%$ & $100.0 \%$ & $100.0 \%$ & $100.0 \%$ & $100.0 \%$ & $100.0 \%$ \\
Depart. EV SOC-100\% & $100.0 \%$ & $100.0 \%$ & $100.0 \%$ & $100.0 \%$ & $100.0 \%$ & $100.0 \%$ \\
\hline
\end{tabular}

the penalty parameters are assigned such that $c_{j, \phi}^{P V}=1.2$, $c_{j, \phi}^{E V}=1$, and $c_{j, \phi}^{H P}=0.8$ throughout the simulation period. While for the HP-priority case, the penalty parameters are assigned as $c_{j, \phi}^{P V}=1.2, c_{j, \phi}^{E V}=0.8$, and $c_{j, \phi}^{H P}=1$. In this way, EVs are given priority for not being curtailed earlier than HPs in the EV-priority case, and vice versa. Impacts on end-users for the above winter case are presented in Table III and IV, respectively. It can be seen from Table II and III that in the EVpriority case, the water tank temperature breaks the lower limit for all scenarios, being $14.9^{\circ} \mathrm{C}, 12.2^{\circ} \mathrm{C}$, and $14.8^{\circ} \mathrm{C}$ lower than their counterparts under the proposed DER curtailment tradeoff strategy. EVs are all fully charged. Under the HP-priority scenario, the minimum water tank temperature is increased by $2.7^{\circ} \mathrm{C}$ under the $50 \%$ DER penetration scenario, while for other scenarios the minimum temperature remains similar. While on the EV side, the minimum charge is $37.4 \%, 50.5 \%$, and $96.6 \%$ for the $50 \%, 75 \%$, and $100 \%$ DER penetration scenarios respectively, which are all lower than the proposed strategy (almost fully charged) and thus affecting end-users' driving comfort. The increase in the minimum EV charge with higher EV penetration levels can be explained by mitigated phase unbalance and thus mitigated undervoltage, as also demonstrated in the study [30].
TABLE IV

END-USERS' COMFORT FOR WINTER CASE UNDER HP-PRIORITY SETTING

\begin{tabular}{lcccccc}
\hline & Min & $\mathrm{Q} 2$ & Median & $\mathrm{Q} 4$ & Max & Mean \\
\hline Tot. PV Curt.(kWh)-50\% & 0.00 & 0.00 & 0.00 & 0.00 & 0.08 & 0.00 \\
Tot. PV Curt.(kWh)-75\% & 0.00 & 0.00 & 0.00 & 0.00 & 0.09 & 0.01 \\
Tot. PV Curt.(kWh)-100\% & 0.00 & 0.00 & 0.00 & 0.01 & 0.13 & 0.01 \\
\hline Min Tank Temp. $\left({ }^{\circ} \mathrm{C}\right)-50 \%$ & 50.26 & 54.93 & 55.00 & 55.00 & 55.00 & 54.82 \\
Min Tank Temp. $\left({ }^{\circ} \mathrm{C}\right)-75 \%$ & 43.75 & 54.30 & 55.00 & 55.00 & 55.00 & 54.42 \\
Min Tank Temp. $\left({ }^{\circ} \mathrm{C}\right)-100 \%$ & 36.16 & 53.41 & 54.49 & 54.92 & 55.00 & 53.38 \\
\hline Depart. EV SOC-50\% & $\mathbf{3 7 . 4 \%}$ & $100.0 \%$ & $100.0 \%$ & $100.0 \%$ & $100.0 \%$ & $99.0 \%$ \\
Depart. EV SOC-75\% & $\mathbf{5 0 . 5 \%}$ & $100.0 \%$ & $100.0 \%$ & $100.0 \%$ & $100.0 \%$ & $99.5 \%$ \\
Depart. EV SOC-100\% & $\mathbf{9 6 . 6 \%}$ & $100.0 \%$ & $100.0 \%$ & $100.0 \%$ & $100.0 \%$ & $99.9 \%$ \\
\hline
\end{tabular}

TABLE V

END-USERS' COMFORT FOR WINTER CASE WITH LINEAR OBJECTIVE

\begin{tabular}{lcccccc}
\hline & Min & Q2 & Median & Q4 & Max & Mean \\
\hline Tot. PV Curt.(kWh)-50\% & 0.00 & 0.00 & 0.00 & 0.00 & 0.00 & 0.00 \\
Tot. PV Curt.(kWh)-75\% & 0.00 & 0.00 & 0.00 & 0.00 & 0.00 & 0.00 \\
Tot. PV Curt.(kWh)-100\% & 0.00 & 0.00 & 0.00 & 0.00 & 0.00 & 0.00 \\
\hline Min Tank Temp. $\left({ }^{\circ} \mathrm{C}\right)-50 \%$ & $\mathbf{4 0 . 0 2}$ & 55.00 & 55.00 & 55.00 & 55.00 & 54.72 \\
Min Tank Temp. $\left({ }^{\circ} \mathrm{C}\right)-75 \%$ & $\mathbf{3 4 . 2 3}$ & 55.00 & 55.00 & 55.00 & 55.00 & 54.34 \\
Min Tank Temp. $\left({ }^{\circ} \mathrm{C}\right)-100 \%$ & $\mathbf{1 0 . 9 0}$ & 55.00 & 55.00 & 55.00 & 55.00 & 53.75 \\
\hline Depart. EV SOC-50\% & $\mathbf{9 2 . 6 \%}$ & $100.0 \%$ & $100.0 \%$ & $100.0 \%$ & $100.0 \%$ & $99.9 \%$ \\
Depart. EV SOC-75\% & $100.0 \%$ & $100.0 \%$ & $100.0 \%$ & $100.0 \%$ & $100.0 \%$ & $100.0 \%$ \\
Depart. EV SOC-100\% & $100.0 \%$ & $100.0 \%$ & $100.0 \%$ & $100.0 \%$ & $100.0 \%$ & $100.0 \%$ \\
\hline
\end{tabular}

TABLE VI

END-USERS' COMFORT FOR WINTER CASE USING RHC APPROACH

\begin{tabular}{lcccccc}
\hline & Min & $\mathrm{Q} 2$ & Median & $\mathrm{Q} 4$ & Max & Mean \\
\hline Tot. PV Curt.(kWh)-50\% & 0.00 & 0.00 & 0.00 & 0.00 & 0.08 & 0.00 \\
Tot. PV Curt.(kWh)-75\% & 0.00 & 0.00 & 0.00 & 0.00 & 0.03 & 0.00 \\
Tot. PV Curt.(kWh)-100\% & 0.00 & 0.00 & 0.00 & 0.00 & 0.02 & 0.00 \\
\hline Min Tank Temp. $\left({ }^{\circ} \mathrm{C}\right)-50 \%$ & 41.92 & 54.75 & 55.00 & 55.00 & 55.00 & 54.29 \\
Min Tank Temp. $\left({ }^{\circ} \mathrm{C}\right)-75 \%$ & 42.65 & 52.52 & 54.99 & 55.00 & 55.00 & 53.30 \\
Min Tank Temp. $\left({ }^{\circ} \mathrm{C}\right)-100 \%$ & $\mathbf{4 0 . 3 0}$ & 49.26 & 51.60 & 54.36 & 55.00 & 51.04 \\
\hline Depart. EV SOC-50\% & $100.0 \%$ & $100.0 \%$ & $100.0 \%$ & $100.0 \%$ & $100.0 \%$ & $100.0 \%$ \\
Depart. EV SOC-75\% & $100.0 \%$ & $100.0 \%$ & $100.0 \%$ & $100.0 \%$ & $100.0 \%$ & $100.0 \%$ \\
Depart. EV SOC-100\% & $100.0 \%$ & $100.0 \%$ & $100.0 \%$ & $100.0 \%$ & $100.0 \%$ & $100.0 \%$ \\
\hline
\end{tabular}

\section{Fairness}

Impacts of the quadratic objective function are assessed in this section, considering end-users' comfort and fairness compared with a linear one which minimizes $\sum_{j \in \mathcal{H}} \sum_{\phi \in \Phi} \Delta_{j, \phi}$. It is noteworthy that $\Delta_{j, \phi}$ is inherently non-negative due to the absolute value settings and constraints (13) and (16). It can be seen from Table $\mathrm{V}$ that the majority of end-users is not affected as seen that the second percentile of the water tank temperature is $55^{\circ} \mathrm{C}$. However, the minimum temperature is $40.0^{\circ} \mathrm{C}, 34.2^{\circ} \mathrm{C}, 10.9^{\circ} \mathrm{C}$ for the $50 \%, 75 \%$, and $100 \%$ DER penetration scenarios respectively, which are respectively $7.6^{\circ} \mathrm{C}, 9.5^{\circ} \mathrm{C}, 25.3^{\circ} \mathrm{C}$ lower than their counterparts in the quadratic case. For EV users, the minimum state of charge of the $50 \%$ DER penetration scenario is $92.6 \%$, which is also lower than the quadratic case. The improved end-users' comfort can be explained by the fairer curtailment, thus not penalizing individual end-user drastically.

Fig. 8 and 9 present respectively aggregated active power curtailment in the network and the fairness indicator using the Jain's fairness index [20], defined in (29), which ranges from 0 to 1 with 1 being the fairest distribution and 0 being the unfairest distribution. In this study, $x_{i}$ represents combined active power curtailment of household $i$. Under all scenarios, it can generally be seen that the quadratic objective enables a fairer curtailment among end-users but ends up 


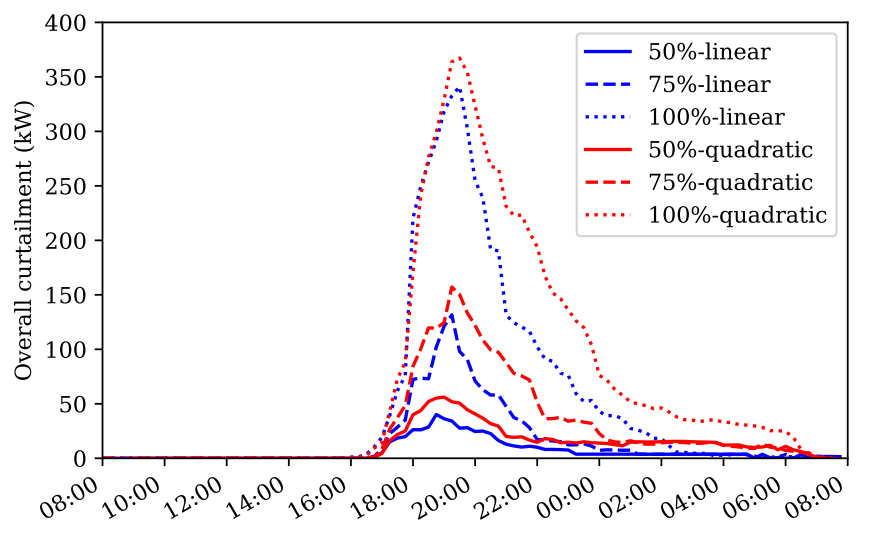

Fig. 8. Aggregated real power curtailment for different objective functions and DER penetration levels.

losing potential active power usage. This is reasonable as the quadratic formulation largely penalizes higher curtailment, thus driving the curtailment from end-users to a similar level. A proof of this fairness improvement from use of the quadratic objective function is provided in the Appendix. Trade-off between more power curtailment and fairness can be decided by DSOs according to local policies. In summary, the quadratic objective function leads to improved end-users' comfort and fairness.

$$
\mathcal{J}\left(x_{1}, x_{2}, \ldots, x_{n}\right)=\frac{\left(\sum_{i=1}^{n} x_{i}\right)^{2}}{n \sum_{i=1}^{n} x_{i}^{2}}=\frac{\overline{\mathbf{x}}^{2}}{\overline{\mathbf{x}^{2}}}
$$

\section{E. Comparison with RHC}

This final section shows the comparison of the proposed strategy with a rolling horizon control approach. The study [16] can be referred to for formulation of the RHC approach. In an overview, the model takes as parameters one-day ahead forecast of base load, PV generation, EV arrival/departure time and energy need, as well as heat demand, and optimizes active and reactive power set-points of PVs, EVs, and HPs during the forecast horizon to ensure network integrity while minimizing impacts on end-users' comfort (i.e. minimizing aggregated PV curtailment, unserved EV demand, and maximum temperature drop over the forecast horizon). To account for uncertainties with a longer-term forecast, a Gaussian distributed forecast error is assumed evolving linearly from $0 \%$ normalized root mean squared error (NRMSE) at the first time step to $30 \%$ at the end of the forecast horizon of 24 hours for PV generation, heat demand, and base load. The EV arrival/departure time and energy demand are assumed known for easier formulation. For both models, network issues have been fully removed. Looking into end-users' comfort in Table VI, it is seen that the RHC model can keep end-users' comfort at the extreme case of $100 \%$ DER penetration, thus presenting better performance to keep end-users' comfort due to its longer forecast horizon. However, the proposed strategy is already able to handle a high DER loading of $75 \%$, and only 2 out of 182 endusers' thermal comfort is affected (going beyond the lower water tank temperature limit by $0.1^{\circ} \mathrm{C}$ and $3.8^{\circ} \mathrm{C}$, respectively) under the $100 \%$ DER penetration scenario. This impact can

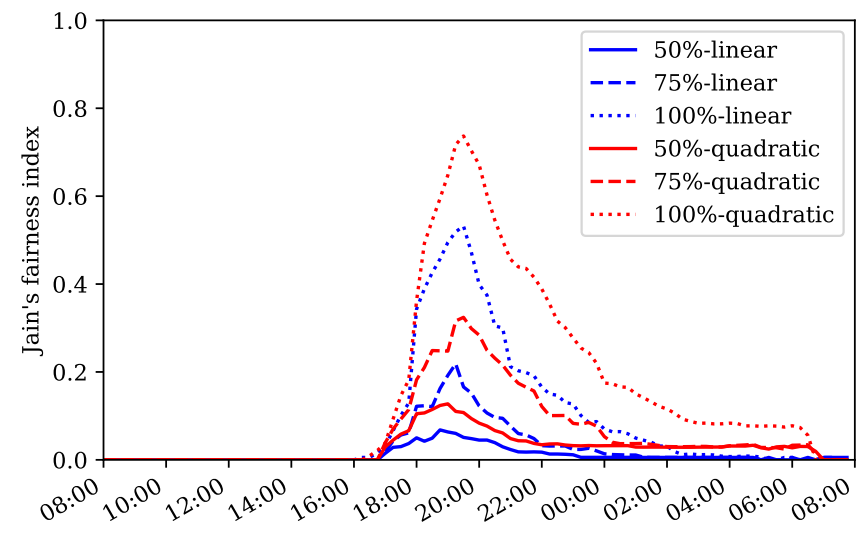

Fig. 9. Jain's fairness index for real power curtailment for different objective functions and DER penetration levels.

be further reduced when additional thermal flexibility from the household's comfort temperature range is considered.

In terms of computational performance, two additional measures have been applied to enhance computation speed of the RHC based model. First, granularity of the forecast is set as 1 hour except the first time step (15 minutes) [16] where control decisions are implemented. Second, the first stage uses only a one time step forecast as voltage accuracy of following time steps is seen as less important than the first one. Using these two measures, memory requirement and time taken for the RHC approach have decreased from around $500 \mathrm{MB}, 385$ seconds to around $130 \mathrm{MB}, 50$ seconds for each time step. In comparison, the proposed single-period optimization approach requires only around $5 \mathrm{MB}$ of memory and 3 seconds to solve. Relief in computation can facilitate minute-scale or secondscale network operations for DSOs as distribution networks are becoming increasingly dynamic with more DERs. When considering a larger network and even integer variables from legacy grid assets or household batteries, computation burden for the RHC approach can further be a problem.

\section{CONCLusions}

A single-period OPF-based strategy is proposed in this paper for DSOs to manage LV grids where increasing DERs with intertemporal constraints such as EVs and HPs are connected. Through a case study, it is found that the proposed strategy can fully resolve network issues while maintaining end-users' comfort, until up to $75 \%$ of DER penetration. Such a single-period optimization is facilitated by the proposed DER curtailment trade-off strategy based on their remaining flexibility. Through a quadratic objective function, the OPF formulation can ensure a fairer curtailment and improved end-users' comfort. The comparison with a rolling horizon control approach found that the proposed strategy can achieve much faster computation and smaller memory requirement, allowing DSOs for minute-scale and even second-scale grid operations, though end-users' comfort is slightly compromised at the extreme loading condition of $100 \%$ DER penetration (water tank temperature is respectively $0.1{ }^{\circ} \mathrm{C}$, and $3.8^{\circ} \mathrm{C}$ lower than the limit for 2 out of 182 end-users). Impacts of the 
proposed strategy can be further tested by DSOs on their grids and served end-users. In conclusion, this work opens new opportunities for DSOs to manage LV grids facing a large amount DERs with intertemporal constraints, which is considered increasingly important in view of the ongoing energy transition and evolution of passive distribution networks to active distribution networks.

\section{APPENDIX}

Let $x^{l i n}$ and $x^{\text {quad }}$ be the solutions to a general optimization problem (30) where $f(x)=\sum_{i=1}^{n} x_{i}$ and $f(x)=\sum_{i=1}^{n} x_{i}^{2}$, respectively.

$$
\begin{array}{cl}
\underset{x}{\operatorname{minimize}} & f(x) \\
\text { subject to } & g_{i}(x) \leq 0, \quad i=1, \ldots, m \\
& h_{j}(x)=0, \quad j=1, \ldots, p \\
& x_{i} \geq 0, \quad i=1, \ldots, n
\end{array}
$$

Following this, we have $0 \leq \sum_{i=1}^{n} x_{i}^{\text {lin }} \leq \sum_{i=1}^{n} x_{i}^{\text {quad }}$ and $0 \leq \sum_{i=1}^{n}\left(x_{i}^{\text {quad }}\right)^{2} \leq \sum_{i=1}^{n}\left(x_{i}^{\text {lin }}\right)^{2}$ from the minimization formulation. Then (31) holds if there exist an $i$ and a $j$ such that $x_{i}^{\text {quad }} \neq 0$ and $x_{j}^{\text {lin }} \neq 0$.

$$
\mathcal{J}\left(x^{\text {quad }}\right)=\frac{\left(\sum_{i=1}^{n} x_{i}^{\text {quad }}\right)^{2}}{n \sum_{i=1}^{n}\left(x_{i}^{\text {quad }}\right)^{2}} \geq \frac{\left(\sum_{i=1}^{n} x_{i}^{\text {lin }}\right)^{2}}{n \sum_{i=1}^{n}\left(x_{i}^{\text {lin }}\right)^{2}}=\mathcal{J}\left(x^{\text {lin }}\right)
$$

\section{REFERENCES}

[1] P. D. Ferreira, P. M. Carvalho, L. A. Ferreira, and M. D. Ilic, "Distributed energy resources integration challenges in low-voltage networks: Voltage control limitations and risk of cascading," IEEE Trans. Sustain. Energy, vol. 4, no. 1, pp. 82-88, 2013.

[2] R. A. Verzijlbergh, M. O. Grond, Z. Lukszo, J. G. Slootweg, and M. D. Ilic, "Network impacts and cost savings of controlled EV charging," IEEE Trans. Smart Grid, vol. 3, no. 3, pp. 1203-1212, 2012.

[3] S. Deilami, A. S. Masoum, P. S. Moses, and M. A. Masoum, "Realtime coordination of plug-in electric vehicle charging in smart grids to minimize power losses and improve voltage profile," IEEE Trans. Smart Grid, vol. 2, no. 3, pp. 456-467, 2011.

[4] G. Benetti, M. Delfanti, T. Facchinetti, D. Falabretti, and M. Merlo, "Real-Time Modeling and Control of Electric Vehicles Charging Processes," IEEE Trans. Smart Grid, vol. 6, no. 3, pp. 1375-1385, 2015.

[5] J. Quirós-Tortós, L. F. Ochoa, S. W. Alnaser, and T. Butler, "Control of EV Charging Points for Thermal and Voltage Management of LV Networks," IEEE Trans. Power Syst., vol. 31, no. 4, pp. 3028-3039, 2016.

[6] I. I. Avramidis, F. Capitanescu, and G. Deconinck, "A Comprehensive Multi-Period Optimal Power Flow Framework for Smart LV Networks," IEEE Trans. Power Syst., vol. 36, no. 4, pp. 3029-3041, 2021.

[7] M. Z. Liu, L. F. Ochoa, and S. H. Low, "On the Implementation of OPF-Based Setpoints for Active Distribution Networks," IEEE Trans. Smart Grid, vol. 12, no. 4, pp. 2929-2940, jul 2021.

[8] E. Dall'Anese, S. V. Dhople, and G. B. Giannakis, "Optimal dispatch of photovoltaic inverters in residential distribution systems," IEEE Trans. Sustain. Energy, vol. 29, no. 4, pp. 957-967, 2014.

[9] E. Dallranese, S. V. Dhople, B. B. Johnson, and G. B. Giannakis, "Optimal dispatch of residential photovoltaic inverters under forecasting uncertainties," IEEE J. Photovoltaics, vol. 5, no. 1, pp. 350-359, 2015.

[10] T. Ding, C. Li, Y. Yang, J. Jiang, Z. Bie, and F. Blaabjerg, "A Two-Stage Robust Optimization for Centralized-Optimal Dispatch of Photovoltaic Inverters in Active Distribution Networks," IEEE Trans. Sustain. Energy, vol. 5, no. 2, pp. 487-497, 2014.
[11] E. Dall'Anese, S. V. Dhople, B. B. Johnson, and G. B. Giannakis, "Decentralized optimal dispatch of photovoltaic inverters in residential distribution systems," IEEE Trans. Energy Convers., vol. 29, no. 4, pp. 957-967, 2014

[12] X. Su, M. A. Masoum, and P. J. Wolfs, "Optimal PV inverter reactive power control and real power curtailment to improve performance of unbalanced four-wire LV distribution networks," IEEE Trans. Sustain. Energy, vol. 5, no. 3, pp. 967-977, 2014.

[13] N. Nazir and M. Almassalkhi, "Voltage positioning using cooptimization of controllable grid assets in radial networks," IEEE Trans. Power Syst., vol. 36, no. 4, pp. 2761-2770, 2021.

[14] J. D. Watson, N. R. Watson, and I. Lestas, "Optimal Scheduling of Battery Energy Storage Systems in Unbalanced Distribution Networks," IEEE Trans. Sustain. Energy, vol. 9, no. 2, pp. 639-650, 2018.

[15] N. Nazir, P. Racherla, and M. Almassalkhi, "Optimal Multi-Period Dispatch of Distributed Energy Resources in Unbalanced Distribution Feeders," IEEE Trans. Power Syst., vol. 35, no. 4, pp. 2683-2692, 2020.

[16] C. Sabillon, J. F. Franco, M. J. Rider, and R. Romero, "Joint optimal operation of photovoltaic units and electric vehicles in residential networks with storage systems: A dynamic scheduling method," Int. J. Electr. Power Energy Syst., vol. 103, no. March, pp. 136-145, 2018.

[17] Y. Guo, Q. Wu, H. Gao, X. Chen, J. Ostergaard, and H. Xin, "MPCBased Coordinated Voltage Regulation for Distribution Networks with Distributed Generation and Energy Storage System," IEEE Trans. Sustain. Energy, vol. 10, no. 4, pp. 1731-1739, 2019.

[18] F. Gangale, A. Mengolini, and I. Onyeji, "Consumer engagement: An insight from smart grid projects in Europe," Energy Policy, vol. 60, pp. 621-628, sep 2013.

[19] O. Ellabban and H. Abu-Rub, "Smart grid customers' acceptance and engagement: An overview," Renew. Sustain. Energy Rev., vol. 65, pp. 1285-1298, nov 2016.

[20] K. Petrou, A. T. Procopiou, L. Gutierrez-Lagos, M. Z. Liu, L. F. Ochoa, T. Langstaff, and J. Theunissen, "Ensuring Distribution Network Integrity Using Dynamic Operating Limits for Prosumers," IEEE Trans. Smart Grid, pp. 1-11, 2021.

[21] K. Petrou, M. Z. Liu, A. T. Procopiou, L. F. Ochoa, J. Theunissen, and J. Harding, "Operating envelopes for prosumers in LV networks: A weighted proportional fairness approach," IEEE PES Innov. Smart Grid Technol. Conf. Eur., vol. 2020-Octob, 2020.

[22] L. Gutierrez-Lagos and L. F. Ochoa, "OPF-Based CVR Operation in PVRich MV-LV Distribution Networks," IEEE Trans. Power Syst., vol. 34, no. 4, pp. 2778-2789, 2019.

[23] J. S. Giraldo, P. P. Vergara, J. C. Lopez, P. H. Nguyen, and N. G. Paterakis, "A Linear AC-OPF Formulation for Unbalanced Distribution Networks," IEEE Trans. Ind. Appl., pp. 1-11, 2021.

[24] L. Gutierrez-Lagos, M. Z. Liu, and L. F. Ochoa, "Implementable ThreePhase OPF Formulations for MV-LV Distribution Networks: MILP and MIQCP," in IEEE PES Conf. Innov. Smart Grid Technol. ISGT Lat. Am., 2019, pp. $1-6$.

[25] S. Zhan, "Research data for a single-period opf-based strategy to manage distributed energy resources with intertemporal constraints in low-voltage grids," 4TU.ResearchData, 2021. [Online]. Available: https://doi.org/10.4121/16822393

[26] Solcast, "Solar irradiance data." [Online]. Available: http://solcast.com

[27] N. Good, E. Karangelos, A. Navarro-Espinosa, and P. Mancarella, "Optimization under Uncertainty of Thermal Storage-Based Flexible Demand Response with Quantification of Residential Users' Discomfort," IEEE Trans. Smart Grid, vol. 6, no. 5, pp. 2333-2342, 2015.

[28] Gurobi Optimization, LLC, "Gurobi optimizer reference manual," 2021. [Online]. Available: http://www.gurobi.com

[29] L. Thurner, A. Scheidler, F. Schafer, J. H. Menke, J. Dollichon, F. Meier, S. Meinecke, and M. Braun, "pandapower - an open source python tool for convenient modeling, analysis and optimization of electric power systems," IEEE Trans. Power Syst., 2018.

[30] M. Gray and W. Morsi, "Probabilistic quantification of voltage unbalance and neutral current in secondary distribution systems due to plug-in battery electric vehicles charging," Electr. Power Syst. Res., vol. 133, pp. 249-256, 2016. 